Proc. of the 11 Int. School on Theoretical Physics Symmetry and Structural Properties of Condensed Matter, Rzeszów 2014

\title{
Exciton Binding Energy and Oscillator Strength in a Shallow Quantum Well in an External Magnetic Field
}

\author{
P. ZIĘBA ${ }^{a, *}$, B. PIEcTKA ${ }^{b}$, J. EUSAKOWSKI $^{b}$ AND I. TRALlE ${ }^{a}$ \\ ${ }^{a}$ Faculty of Mathematics and Natural Sciences, University of Rzeszów, 35-310 Rzeszów, Poland \\ ${ }^{b}$ Institute of Experimental Physics, Faculty of Physics, University of Warsaw, \\ L. Pasteura 5, 02-093 Warszawa, Poland
}

\begin{abstract}
We discuss the influence of an external magnetic field on the exciton energy and the exciton oscillator strength in the shallow quantum wells. We include into consideration the Coulomb attraction between electron and hole, which is rarely taken into account. We self-consistently solve the Schrödinger equation to compare the obtained results with the experimental values.
\end{abstract}

DOI: 10.12693 /APhysPolA.128.237

PACS: 71.35.Ji, 75.75.-c, 73.21.Fg, 62.20.-x

\section{Introduction}

The excitons in quantum wells (QWs) form nowadays the basic system of many optoelectronic devices. Our work was triggered by the recent interest in the excitonpolariton physics, where excitons in quantum wells are strongly coupled to semiconductor microcavity mode [1] After pioneering work showing the non-linear effect in this system [2], researchers become interested in the application of external electric and magnetic fields that modifies the excitonic counterpart of the polariton quasiparticle. In this paper we show the influence of external magnetic field on the exciton energy and the exciton oscillator strength, that is a measure of the excitonphoton coupling strength. We are especially interested in excitons in shallow QW reported in the recent publications $[3,4]$. We directly compare our theoretical model with the experimental results reported in Ref. [5].

\section{Exciton energy and oscillator strength}

Coulomb attraction between the electron and hole is responsible for arising an exciton in semiconductors. In the case of QW, the Coulomb force also influences a potential of the QW which localizes particles making the exciton. This type of a QW potential change is rarely taken into account because it is believed to be small in the case of a deep QW. However, in the case considered in this paper, when the material of the quantum well contains only a small fraction of indium, the QW is shallow, and the effect mentioned above should be taken into account. One of the way to deal with it is to solve the exciton problem self-consistently. Here, we follow the method proposed in Ref. [5] and extend it to include the effect of an external magnetic field.

* corresponding author; e-mail: pzieba@if.univ.rzeszow.pl
Let us assume that $z$ axis of the Cartesian coordinates is along the growth direction of heterostructure and suppose that the magnetic field $B$ is normal to the QW plane. Carrying out a standard procedure of separating the exciton center of mass and electron-hole relative motion in the well plane, the heavy hole exciton Hamiltonian can be given in the form

$$
\widehat{H} \Psi=\left(\widehat{H}_{e}+\widehat{H}_{h}+\widehat{H}_{\rho}+V_{c}\right) \Psi=E \Psi,
$$
where

$$
\begin{aligned}
\widehat{H}_{e} & =-\frac{\hbar^{2}}{2 m_{e}} \frac{\partial^{2}}{\partial z_{e}^{2}}+U_{e}\left(z_{e}\right)+\left(l_{e}+1 / 2\right) \hbar \omega_{c}^{e}, \\
\widehat{H}_{h} & =-\frac{\hbar^{2}}{2 m_{h}} \frac{\partial^{2}}{\partial z_{h}^{2}}+U_{h}\left(z_{h}\right)+\left(l_{h}+1 / 2\right) \hbar \omega_{c}^{h}, \\
\widehat{H}_{\rho} & =-\frac{\hbar^{2}}{2 \mu}\left(\frac{1}{\varrho} \frac{\partial}{\partial \varrho}\left(\rho \frac{\partial}{\partial \rho}\right)-\frac{\varrho^{2}}{4 L^{4}}\right), \\
V_{c} & =-\frac{1}{4 \pi \varepsilon_{0} \varepsilon} \frac{e^{2}}{\sqrt{\varrho^{2}+\left(z_{e}-z_{h}\right)^{2}}} .
\end{aligned}
$$

$z_{e}, z_{h}$ are electron and hole $z$-coordinate (along the growth direction), $\rho$ is the in-plane ( $x, y$-plane) electronhole pair separation, $m_{e}, m_{h}$ are the electron and hole masses, $\mu$ - the reduced mass of the electron-hole pair, $\varepsilon_{0}$ is the vacuum permittivity, $e$ is the elementary charge, $\varepsilon$ is the dielectric constant, $L=\sqrt{\hbar /(e B)}$ is the magnetic length, $\omega_{c}^{e}, \omega_{e}^{h}$ are the electron and hole cyclotron frequencies, $U_{e}\left(z_{e}\right), U_{h}\left(z_{h}\right)$ are the well potentials for an electron and a hole, $l_{e}, l_{h}$ electron and hole numbers Landau levels. We assume the exciton wave function to be

$$
\Psi\left(z_{e}, z_{h}, \rho\right)=\phi_{e}\left(z_{e}\right) \phi_{h}\left(z_{h}\right) \psi_{\rho}(\rho) .
$$

To solve Eq. (1) self-consistently we have to find a solution to the following system of integro-differential equations [6]:

$$
\begin{aligned}
& \left(\widehat{H}_{e}+\bar{V}_{e}\right) \phi_{e}\left(z_{e}\right)=E_{e} \phi_{e}\left(z_{e}\right), \\
& \left(\widehat{H}_{h}+\bar{V}_{h}\right) \phi_{h}\left(z_{h}\right)=E_{h} \phi_{h}\left(z_{h}\right), \\
& \left(\widehat{H}_{\rho}+\bar{V}_{\rho}\right) \psi_{\rho}(\rho)=E_{x} \psi_{\rho}(\rho),
\end{aligned}
$$

with effective potentials 


$$
\begin{aligned}
\bar{V}_{e}\left(z_{e}\right) & =\iint \psi_{\rho}^{\star}(\rho) \phi_{h}^{\star}\left(z_{h}\right) V_{c} \psi_{\rho}(\rho) \phi_{h}\left(z_{h}\right) \mathrm{d} z_{h} \mathrm{~d} \rho, \\
\bar{V}_{h}\left(z_{h}\right) & =\iint \psi_{\rho}^{\star}(\rho) \phi_{e}^{\star}\left(z_{e}\right) V_{c} \psi_{\rho}(\rho) \phi_{e}\left(z_{e}\right) \mathrm{d} z_{e} \mathrm{~d} \rho, \\
\bar{V}_{\rho}(\rho) & =\iint \phi_{e}^{\star}\left(z_{e}\right) \phi_{h}^{\star}\left(z_{h}\right) V_{c} \phi_{e}\left(z_{e}\right) \phi_{h}\left(z_{h}\right) \mathrm{d} z_{e} \mathrm{~d} z_{h},
\end{aligned}
$$

and with total energy $E$ is given by

$$
\begin{aligned}
E & =E_{e}+E_{h}+E_{x}-\int \phi_{e}^{\star}\left(z_{e}\right) \bar{V}_{e} \phi_{e}\left(z_{e}\right) \mathrm{d} z_{e} \\
& -\int \phi_{h}^{\star}\left(z_{h}\right) \bar{V}_{h} \phi_{h}\left(z_{h}\right) \mathrm{d} z_{h} .
\end{aligned}
$$

The system (7)-(9) is solved by the following iteration procedure. First, we solve Eqs. (7) and (8) by setting $\bar{V}_{e}\left(z_{e}\right)=\bar{V}_{h}\left(z_{h}\right)=0$. The obtained solutions $\phi_{e}\left(z_{e}\right)$ and $\phi_{h}\left(z_{h}\right)$ are used to calculate $\bar{V}_{\rho}(\rho)$ by using (12). This in turn enables us to solve Eq. (9). The obtained function $\psi_{\rho}(\rho)$ is then substituted to (10) and (11) to get new approximations of the potentials $\bar{V}_{e}\left(z_{e}\right), \bar{V}_{h}\left(z_{h}\right)$. The procedure is continued until the potentials (10)-(12) become consistent with each other

$$
\begin{gathered}
\int \phi_{e}^{\star}\left(z_{e}\right) \bar{V}_{e} \phi_{e}\left(z_{e}\right) \mathrm{d} z_{e} \approx \int \phi_{h}^{\star}\left(z_{h}\right) \bar{V}_{h} \phi_{h}\left(z_{h}\right) \mathrm{d} z_{h} \approx \\
\int \psi_{\rho}^{\star}(\rho) \bar{V}_{r} \psi_{\rho}(\rho) \mathrm{d} \rho .
\end{gathered}
$$

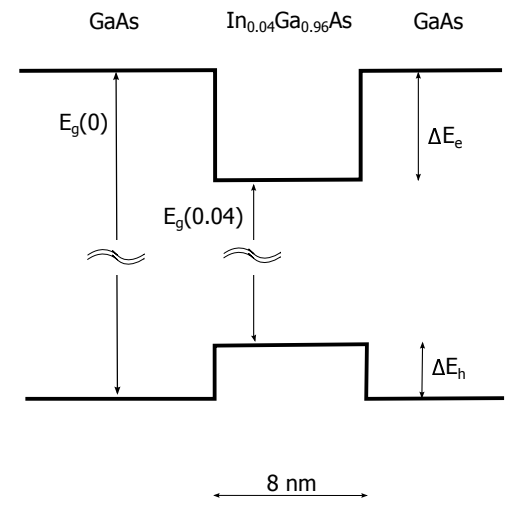

Fig. 1. The quantum well.

In order to solve the problem defined above, we have to estimate QW potentials $U_{e}\left(z_{e}\right), U_{h}\left(z_{h}\right)$ for an electron and a hole. The $\mathrm{In}_{0.04} \mathrm{Ga}_{0.96}$ As quantum well of the width of $8 \mathrm{~nm}$ (see Fig. 1) is supposed to be in between two GaAs barriers. Let us estimate barrier heights of confining potentials for a heavy hole and an electron. Typically, they are determined by a difference in band gaps shared between the conduction and valence bands according to a factor $Q$ which is the ratio of the conduction to valence band offsets $\Delta E_{c}: \Delta E_{v}$. In the case of the quantum well under consideration, there is a lattice mismatch between materials of the well and the barriers. The lattice constant of $\mathrm{In}_{0.04} \mathrm{Ga}_{0.96} \mathrm{As}$ is larger than that of GaAs which causes a compressive strain of the QW, which in its turn introduces an additional contribution to the confining potential. The strain tensor is

$$
\epsilon=\left[\begin{array}{ccc}
\epsilon_{x x} & 0 & 0 \\
0 & \epsilon_{y y} & 0 \\
0 & 0 & \epsilon_{z z}
\end{array}\right],
$$

where

$$
\epsilon_{x x}=\epsilon_{y y}=\delta, \quad \epsilon_{z z}=-2 \delta \frac{c_{12}}{c_{11}}, \quad \delta=\frac{a_{x}-a_{0}}{a_{x}},
$$

$x$ is indium atomic content, $a_{x}$ - an $x$-dependent lattice constant of $\operatorname{In}_{x} \mathrm{Ga}_{1-x} \mathrm{As}$, and $c_{12}, c_{11}$ are the elastic stiffness constants. The energy shifts due to the strain for the conduction and heavy hole valence band edges are

$$
\begin{aligned}
& \Delta E_{c}^{\text {strain }}=a_{c}\left(\epsilon_{x x}+\epsilon_{y y}+\epsilon_{z z}\right), \\
& \Delta E_{v}^{\text {strain }}=a_{v}\left(\epsilon_{x x}+\epsilon_{y y}+\epsilon_{z z}\right)-b_{v}\left(\epsilon_{x x}-\epsilon_{z z}\right),
\end{aligned}
$$

where $a_{c}, a_{v}, b_{v}$ are deformation potentials for the heavy hole valence and conduction bands, respectively. An $x$ dependence of the band gap in the unstrained case is [7]:

$$
E_{g}(x)=E_{g}(0)-1.5837 x+0.475 x^{2} .
$$

The total shift of the band is

$$
\Delta E=1.5837 x-0.475 x^{2}-\Delta E_{v}^{\text {strain }}-\Delta E_{c}^{\text {strain }} .
$$

To account for temperature changes in the lattice constant and band gaps we use the following formulae:

$$
\begin{aligned}
& a(T)=a(300 \mathrm{~K})+(T-300 \mathrm{~K}) a_{T}, \\
& E_{g}(T)=E_{g}(0)-\frac{\alpha T^{2}}{T+\beta},
\end{aligned}
$$

$\alpha$ and $\beta$ are the material constants. To obtain the parameters of $\operatorname{In}_{x} \mathrm{Ga}_{1-x} \mathrm{As}$ for the indium fraction $x=0.04$, we use a linear interpolation of parameters of GaAs and InAs given in Table. Assuming $\mathrm{Q}=60: 40$ [8], we get $\Delta E_{h h}$ $=18 \mathrm{meV}$ and $\Delta E_{e}=\Delta E_{g}-\Delta E_{h h}=27 \mathrm{meV}$. These values determine the confining potentials $U_{e}\left(z_{e}\right), U_{h}\left(z_{h}\right)$.

Material parameters used in calculations.

TABLE

\begin{tabular}{c|c|c}
\hline \hline Parameter & GaAs & InAs \\
\hline$a_{c}[\mathrm{eV}][9]$ & -9.36 & -6.66 \\
$a_{v}[\mathrm{eV}][9]$ & -1.21 & -1.00 \\
$b_{v}[\mathrm{eV}][9]$ & -2.0 & -1.8 \\
$c_{11}[\mathrm{GPa}]$ & 122.1 & 83.29 \\
$c_{12}[\mathrm{GPa}]$ & 56.6 & 45.26 \\
$m_{c}\left[m_{e}\right][10]$ & 0.067 & 0.026 \\
$m_{v}\left[m_{e}\right][10]$ & 0.5 & 0.041 \\
$E_{g}[\mathrm{eV}], T=0 \mathrm{~K}[10]$ & 1.519 & 0.417 \\
$a[\AA] T=300 \mathrm{~K}[10]$ & 5.65325 & 6.0583 \\
$\varepsilon[1][7]$ & 12.93 & 15.15 \\
$\alpha[\mathrm{meV} / \mathrm{K}][10]$ & 540.5 & 276.0 \\
$\beta[\mathrm{K}][10]$ & 204 & 93 \\
$a_{T}[\AA / \mathrm{K}][10]$ & $3.88 \times 10^{-5}$ & $2.74 \times 10^{-5}$
\end{tabular}

The dependence of the calculated exciton energy on the magnetic field is presented in Fig. 2.

It is worth mentioning that we present here results of the calculations for $l_{e}$ and $l_{h}$ which are equal to zero. We also calculate an exciton oscillator strength which is a measure of the probability of an electric dipole optical transitions. It is given by [11]: 


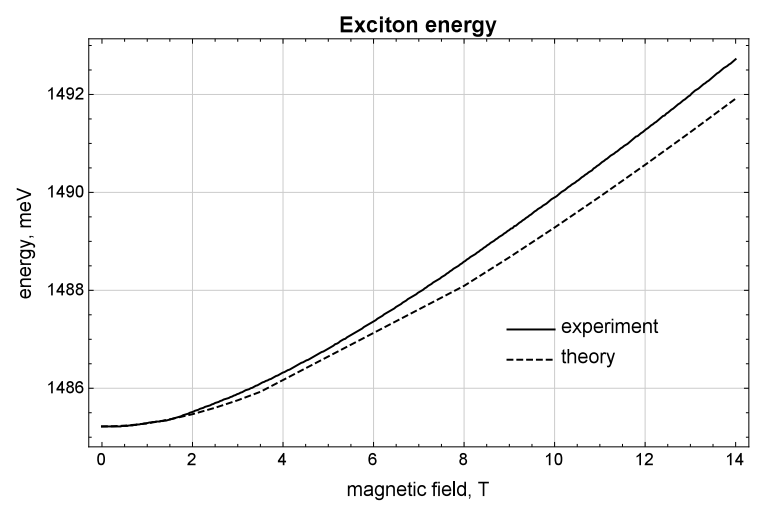

Fig. 2. The exciton energy.

$$
f_{\text {osc }} \propto\left|\iint \phi_{e}\left(z_{e}\right) \phi_{h}\left(z_{h}\right) \mathrm{d} z_{e} \mathrm{~d} z_{h}\right|^{2}\left|\psi_{\rho}(\rho=0)\right|^{2} .
$$

The dependence of the calculated oscillator strength on the magnetic field is presented in Fig. 3.

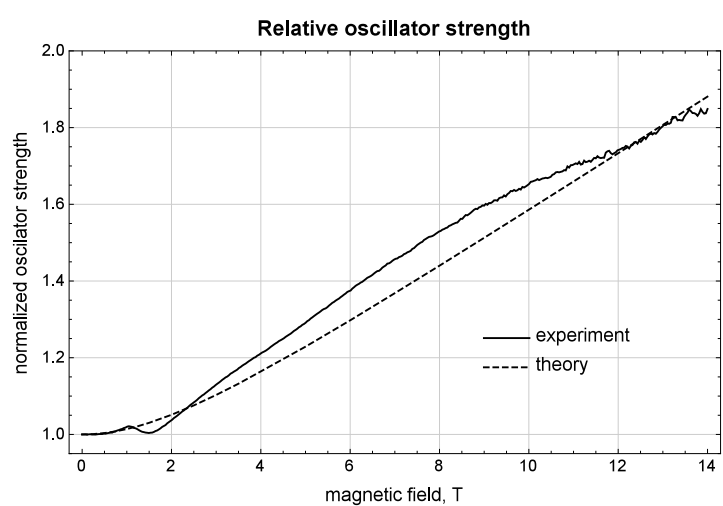

Fig. 3. The normalized oscillator strength..

\section{Conclusions}

Presented results display a sensible agreement between theory and experiment. At low values of the magnetic field when the Landau quantization energy is comparable to the exciton binding energy, we observe a little growth of the normalized oscillator strength. When the magnetic field becomes stronger and the contribution to the exciton energy due to the Landau quantization begins to be significant the oscillator strength grows almost linearly.

\section{Acknowledgments}

The authors acknowledge the support from the National Science Center (NCN) grant 2011/01/D/ST7/04088. I.T. and P.Z. also acknowledge a support from the Centre for Innovation and Transfer of Natural Sciences and Engineering Knowledge at the University of Rzeszów. B.P. would like to thank B. Deveaud for providing the sample and M. Potemski for the help in the experimental work and stimulating discussions.

\section{References}

[1] C. Weisbuch, M. Nishioka, A. Ishikawa, Y. Arakawa, Phys. Rev. Lett. 69, 3314 (1992).

[2] J. Kasprzak, M. Richard, S. Kundermann, A. Baas, P. Jeambrun, J.M.J. Keeling, F.M. Marchetti, M.H. Szymanska, R. André, J.L. Staehli, V. Savona, P.B. Littlewood, B. Deveaud, Le Si Dang, Nature 443 , 409 (2006).

[3] G. Nardin, G. Grosso, Y. Léger, B. Pietka, F. MorierGenoud, B. Deveaud-Plédran, Nature Phys. 7, 635 (2011).

[4] G. Nardin, Y. Léger, B. Piętka, F. Morier-Genoud, B. Deveaud-Plédran, Phys. Rev. B 82, 045304 (2010).

[5] B. Piętka, D. Zygmunt, M. Król, J. Szczytko, J. Łusakowski, M.R. Molas, A.A.L. Nicolet, M. Potemski, P. Stępnicki, M. Matuszewski, P. Zięba, I. Tralle, F. Morier-Genoud, B. Deveaud, Physical Review B 91, 075309 (2015).

[6] I.V. Ponomarev, L.I. Deych, V.A. Shuvayev, A.A. Lisyansky, Physica E 25, 539 (2005).

[7] H. Landolt, R. Börnstein, H. Fischer, O. Madelung, G. Deuschle, Landolt-Börnstein: Numerical Data and Functional Relationships in Science and Technology, Springer-Verlag, Berlin 1987.

[8] D.J. Arent, K. Deneffe, C. Van Hoof, J. De Boeck, G. Borghs, J. Appl. Phys. 66, 1739 (1989).

[9] S.-H. Wei, A. Zunger, Appl. Phys. Lett. 72, 2011 (1998).

[10] I. Vurgaftman, J.R. Meyer, L.R. Ram-Mohan, J. Appl. Phys. 89, 5815 (2001).

[11] L.C. Andreani, in: Proc. Int. School of Physics "Enrico Fermi", Eds. B. Deveaud, A. Quattropani, Vol. 150, IOS Press, Amsterdam 2003, p. 105. 